\title{
Fetal Echocardiographic Assessment of Endocardial Fibroelastosis in Maternal Anti-SSA Antibody-Associated Complete Heart Block
}

\author{
Hisaaki Aoki, MD; Noboru Inamura, MD, PhD; Yukiko Kawazu, MD, PhD; \\ Masahiro Nakayama, MD; Futoshi Kayatani, MD
}

\begin{abstract}
Background: There are few reports describing the features of maternal anti-SSA antibody-associated congenital complete heart block (CCHB) patients developing endocardial fibroelastosis (EFE). The aim of this study was to describe the clinical features and the outcome of patients with $\mathrm{CCHB}$, with or without EFE.
\end{abstract}

\begin{abstract}
Methods and Results: Over a 20-year period, 12 consecutive patients diagnosed with maternal anti-SSA antibody-associated CCHB were identified. The maternal anti-SSA antibody levels were measured and fetal echocardiographic findings were reviewed. The ratios of the thickness of the endocardium to that of the whole wall of the left ventricle (LE/W) and right ventricle (RE/W) were measured to investigate the degree of endocardial thickening. A total of 7 patients survived (living group) and were not diagnosed as having EFE. The remaining 5 patients died and were diagnosed with EFE during autopsy (dead group). Fetal echocardiography of the patients showed differences in the thickening and hyperintensity of the endocardium. The RE/W value was significantly higher in the dead group than in the living group. The titers of both maternal anti-52-kDa and anti-60-kDa SSA antibodies were high, but showed no significant differences between the 2 patient groups.
\end{abstract}

Conclusions: EFE was the major negative prognostic factor for $\mathrm{CCHB}$. Myocardial damage, predominantly in the right ventricle, was related to the outcome of CCHB associated with EFE. (Circ J 2011; 75: 1215-1221)

Key Words: Anti-SSA antibody; Congenital complete heart block; Endocardial fibroelastosis; Fetal echocardiography

$\mathbf{M}$ aternal anti-SSA antibody is associated with congenital complete heart block (CCHB)., ${ }^{1,2}$ Previous reports have described endocardial fibroelastosis (EFE) or other cardiomyopathies as being associated with maternal anti-SSA antibody-mediated CCHB. Both EFE and anti-SSA antibody-mediated CCHB result in progressive congestive heart failure. ${ }^{3,4}$ When EFE is associated with CCHB, there is a very high mortality rate, ${ }^{5}$ however, the etiology of EFE associated with CCHB remains unclear. Some patients with $\mathrm{CCHB}$ die in the perinatal period. It is assumed that they would have also eventually developed EFE. There are, however, few reports that have described patients diagnosed with CCHB, with or without EFE. This study aims to describe the clinical features of patients with maternal antiSSA antibody-associated CCHB with or without EFE by fetal echocardiography, and attempts to clarify the outcomes of the condition.

\begin{abstract}
Methods
Patients

Between 1985 and 2005, 12 consecutive patients who were diagnosed with maternal anti-SSA antibody-associated $\mathrm{CCHB}$ during the fetal period were identified. They were born to mothers positive for anti-SSA antibodies at the Osaka Medical Center and Research Institute for Maternal and Child Health, Izumi, Osaka, Japan. Pre- and postnatal medical records were reviewed for clinical parameters, including perinatal outcome.
\end{abstract}

\section{Fetal Echocardiography}

Fetal echocardiography with cardiovascular anatomical assessment was performed according to techniques previously described $^{6}$ using a variety of echocardiography systems (Advanced Technology Laboratories, Bothell, WA, USA). All images had been recorded on videotape for offline analy-

Received October 14, 2010; revised manuscript received January 5, 2011; accepted January 26, 2011; released online March 22, 2011 Time for primary review: 32 days

Department of Pediatric Cardiology (H.A., N.I., Y.K., F.K.), Department of Pathology (M.N.), Osaka Medical Center and Research Institute for Maternal and Child Health, Izumi, Osaka, Japan

Grant: none.

Mailing address: Hisaaki Aoki, MD, Department of Pediatrics, Sakai Municipal Hospital, 1-1-1 Minamiyasuicho, Sakai-ku, Sakai 590-0078,

Japan. E-mail: aokihisaaki-osk@umin.ac.jp

ISSN-1346-9843 doi:10.1253/circj.CJ-10-1032

All rights are reserved to the Japanese Circulation Society. For permissions, please e-mail: cj@j-circ.or.jp 


\begin{tabular}{|c|c|c|c|c|c|c|c|c|c|c|}
\hline $\begin{array}{l}\text { Case } \\
\text { no. }\end{array}$ & $\begin{array}{c}\text { GA at Dx } \\
\text { (week) }\end{array}$ & $\begin{array}{c}\text { FHR } \\
\text { (beats/min) }\end{array}$ & Hydrops & Treatment & Follow up & $\begin{array}{l}\text { Timing } \\
\text { of PMI }\end{array}$ & Arrhythmia & $\begin{array}{c}\text { Anti-SSA } \\
\text { antibody } \\
\text { (index) }\end{array}$ & Specimen & Prognosis \\
\hline 1 & 18 & 66 & - & ND & 24 years & 3 years & - & 58.8 & ND & Living \\
\hline 2 & 35 & 40 & - & ND & 24 years & 1 days & $\mathrm{R}$ on $\mathrm{T}$ & 651.8 & ND & Living \\
\hline 3 & 36 & 65 & - & ND & 22 years & 4 years & - & 256 & ND & Living \\
\hline 4 & 25 & 40 & - & ND & 15 years & 3 days & - & 32 & ND & Living \\
\hline 5 & 22 & 63 & - & ND & 11 years & 18 days & - & 8 & ND & Living \\
\hline 6 & 31 & 44 & - & ND & 8 years & 2 month & - & 16 & INVM & Living \\
\hline 7 & 23 & 53 & + & Steroid $/ \beta$ & 3 years & 8 days & - & 32 & ND & Living \\
\hline 8 & 37 & 67 & + & Steroid & 15 years & 6 years & $V f$ & 256 & EFE & Dead (15 years old) \\
\hline 9 & 20 & 50 & + & Steroid & 2 years & 1 year & SVT & 256 & EFE & Dead (2 years old) \\
\hline 10 & 22 & 46 & + & ND & 5 days & 0 day & - & 256 & EFE & Dead (neonatal) \\
\hline 11 & 21 & 76 & + & ND & 1 day & ND & VT & 8 & EFE & Dead (neonatal) \\
\hline 12 & 18 & 55 & + & Steroid $/ \beta$ & - & ND & - & 64 & EFE & IUFD \\
\hline
\end{tabular}

GA at Dx, gestational age at diagnosis; FHR, fetal heart rate; PMI, pacemaker implantation; ND, not done; INVM, isolated noncompaction of the ventricular myocardium; $\beta, \beta$ agonist; Vf, ventricular fibrillation; EFE, endocardial fibroelastosis; SVT, supraventricular tachycardia; VT, ventricular tachycardia; IUFD, intrauterine fetal death.

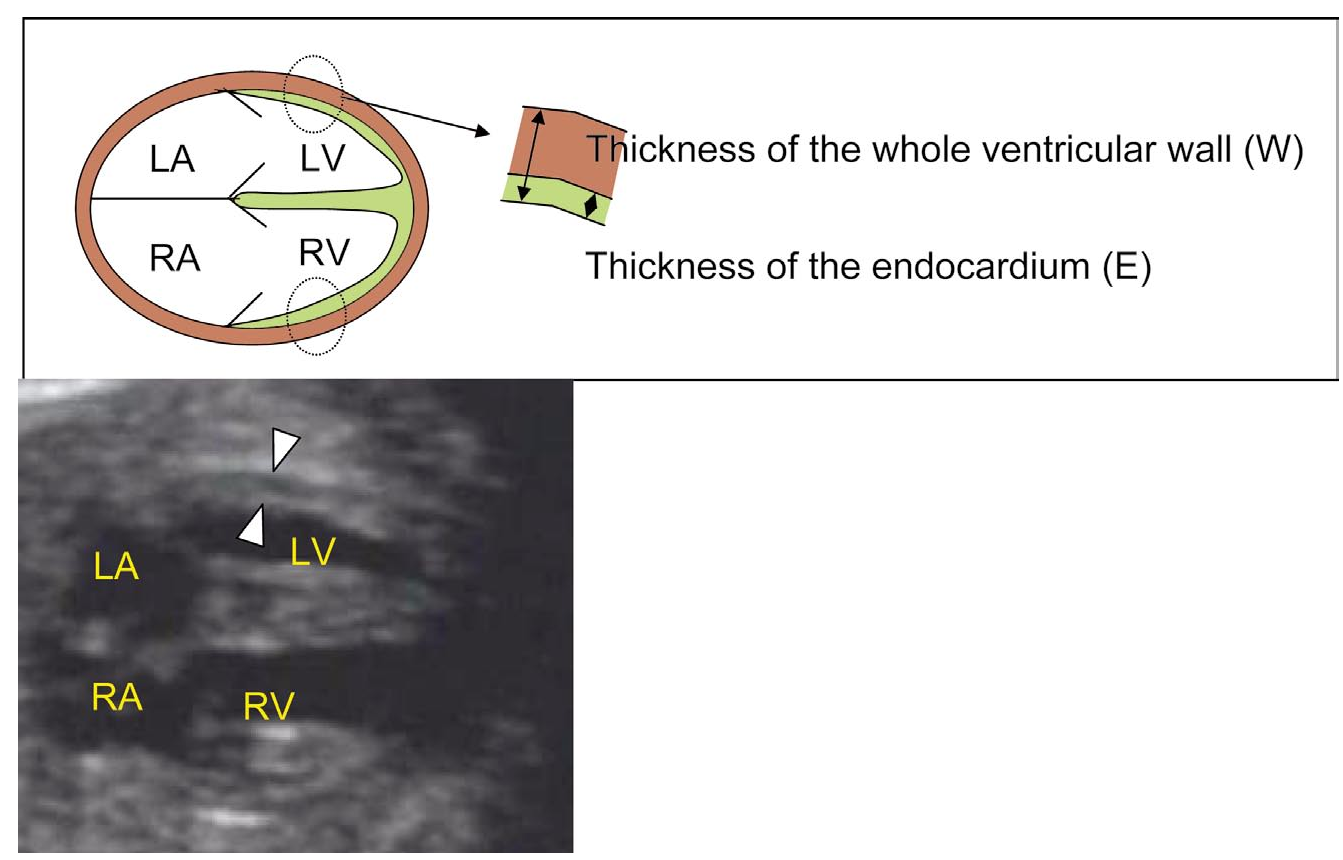

Figure 1. Method of measurement of LE/W and RE/W. The ratio of the thickness of the left and right ventricular endocardium to that of the whole ventricular wall in end-diastole (LE/W; RE/W).

sis. We reviewed fetal echocardiographic findings in 7 (cases $4,5,6,7,9,11$, and 12 of Table 1 ) of the 12 patients whose videotapes were available for analysis. The following dimensions were defined: (1) cardiothoracic area ratio (CTAR); (2) left ventricular fractional shortening (LVFS) and right ventricular fractional shortening (RVFS); and (3) the ratio of the thickness of the ventricular endocardium to that of the whole ventricular wall in the end-diastole of the left (LE/W) and right $(\mathrm{RE} / \mathrm{W})$ ventricles (Figure 1). As a control group, we reviewed similar fetal echocardiographic data from 20 subjects without structural or functional heart disease. Data from echocardiographic studies conducted at the gestational age of approximately 30 weeks were included. The study participants were thus divided into 3 groups: living, dead, and control.

\section{Maternal Autoimmune Antibody}

Anti-SSA antibodies were measured in the mothers of the 12 patients. Two different proteins of $52-\mathrm{kDa}$ and $60-\mathrm{kDa}$ appeared to be the major components of the SSA antigen system. Antibodies to the 52-kDa SSA and 60-kDa SSA antigens were evaluated using enzyme-linked immunosorbent assay (ELISA) techniques with the MBL MESACUP 52k SS-A/ Ro test and the MBL MESACUP 60k SS-A/Ro test, respec- 

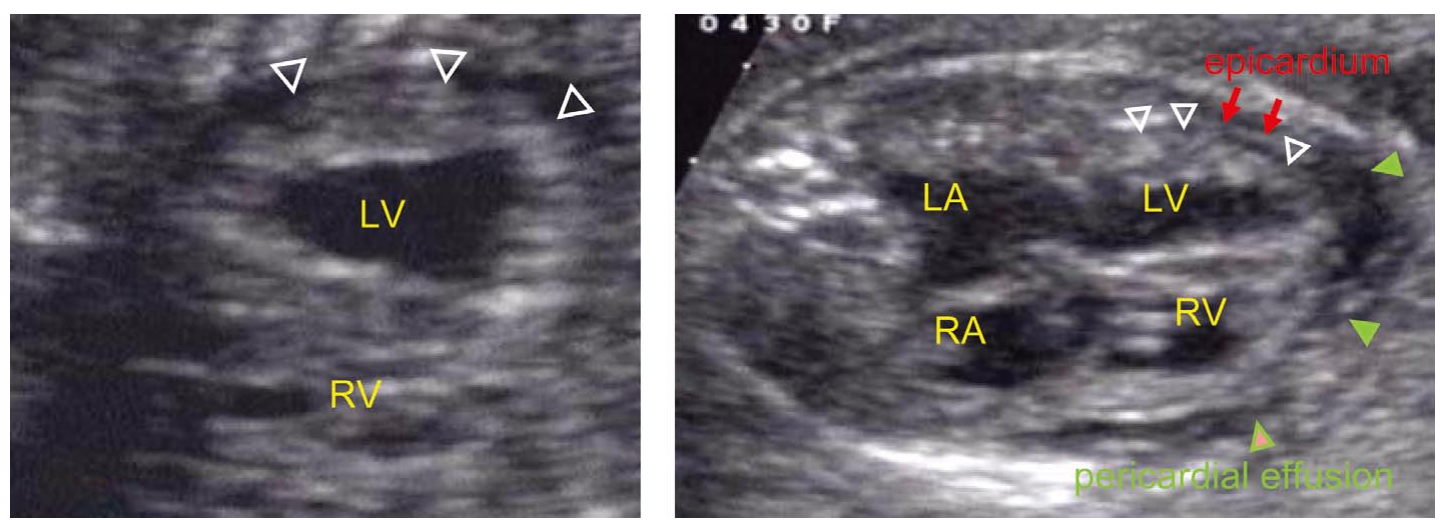

Figure 2. Fetal echocardiographic findings of case 12 with endocardial fibroelastosis (EFE). Thickened and hyperintense endocardium of both ventricles in the short-axis and 4-chamber views, respectively (open arrows). LV, left ventricle; LA, left atrium; $\mathrm{RV}$, right ventricle; $\mathrm{RA}$, right atrium.

\begin{tabular}{|c|c|c|c|c|c|c|c|}
\hline & Control $(n=20)$ & Living ( $n=4)$ & Dead $(n=3)$ & $P$ value & C vs. L & C vs. D & L vs. D \\
\hline CTAR (\%) & $29.1(11.8-34.1)$ & $33.9(36.7-43.1)$ & $41.0(23.3-45.2)$ & $<0.01$ & $<0.01$ & $<0.01$ & NS \\
\hline FHR (beats/min) & $144(130-160)$ & $48(40-63)$ & $55(46-76)$ & $<0.01$ & $<0.01$ & $<0.01$ & NS \\
\hline RVFS (\%) & $27.6(12.6-44.2)$ & $33.3(19.5-41.3)$ & $20.7(12.6-21.9)$ & NS & & & \\
\hline LVFS (\%) & $36.8(25.1-63.3)$ & $48.9(34.7-66.2)$ & $35.1(33.8-40.6)$ & NS & & & \\
\hline RE/W & $0.352(0.143-0.618)$ & $0.241(0.116-0.396)$ & $0.789(0.774-0.907)$ & 0.02 & NS & $<0.01$ & $<0.01$ \\
\hline LE/W & $0.228(0.114-0.571)$ & $0.485(0.174-0.759)$ & $0.649(0.643-0.873)$ & 0.02 & 0.01 & $<0.01$ & NS \\
\hline
\end{tabular}

Regarding echocardiographic data, comparisons between groups were performed using the Kruskal-Wallis nonparametric test. If the overall test was found to be significant, further comparison between the groups of interest was based on the Scheffé test.

CTAR, cardiothoracic ratio; FHR, fetal heart rate; RVFS, right ventricular fractional shortening; LVFS, left ventricular fractional shortening; $\mathrm{RE} / \mathrm{W}$, right ventricular endocardium/whole ventricular wall; LE/W, left ventricular endocardium/whole ventricular wall.

tively. Recombinant human 52-kDa SSA/Ro and 60-kDa SSA/Ro proteins were used as antigens for ELISA. Readings below 5.5 and 3.2 indices for 52-kDA SSA and 60-kDa SSA antibodies, respectively, were considered negative. Correlations were analyzed between the titer of each autoantibody and the prognosis, as well as fetal echocardiographic data including RE/W and LE/W.

\section{Statistical Analysis}

Comparisons between the echocardiographic data groups were performed using the Kruskal-Wallis nonparametric test. If the overall test result was found to be significant, further comparison between the groups of interest was based on the Scheffé test. The Mann-Whitney U-test was used for comparisons regarding anti-SSA antibody titers. A P value of $<0.05$ was considered significant.

\section{Results}

\section{Patients' Profiles}

Clinical data of the 12 patients are summarized in Table 1. Patient follow-up periods ranged from 0 days to 24 years, with a median of 11 years. Seven of the 12 patients survived and were not diagnosed with EFE. One patient was diagnosed by echocardiography as having isolated non-compaction of the ventricular myocardium (INVM). This patient was diagnosed by myocardial biopsy to have myocardial damage. The remaining 5 subjects died (time of death: intrauterine,
1 day, 5 days, 3 years, and 15 years), and the mortality rate was $42 \%$. All patients who died were diagnosed with EFE during autopsy. Three of these 5 patients died without having pacemaker implantation during the perinatal period. Pacemakers were implanted between 0 days and 6 years of age in the remaining 2 patients. Six patients among the total 12 patients developed hydrops fetalis and 4 of them were treated prenatally with steroids and/or a $\beta$ agonist. One patient (case 12) with an extremely deteriorated cardiac performance did not improve, despite administration of a $\beta$ agonist during the fetal period.

\section{Fetal Echocardiographic Findings}

Figure 1 shows the method used for measuring the thickness of the ventricular endocardium and the ventricular wall. Parameters were measured in the free wall of each ventricle. Figure 2 shows the thickened and hyperintense endocardium of both ventricles (arrows). Table 2 shows the correlation between the prognosis and the fetal echocardiographic findings. The values of CTAR, fetal heart rate, and LE/W were significantly higher in the living and dead groups than in controls. However, there was no significant difference between the living and dead groups. Both RVFS and LVFS did not differ significantly among the 3 groups. The value for RE/W was significantly higher in the dead group than in the living group or controls. Figure 3A shows the association between $\mathrm{RE} / \mathrm{W}$ and LE/W. Patients in the dead group (located in the right upper quadrant) showed high values for both; how- 


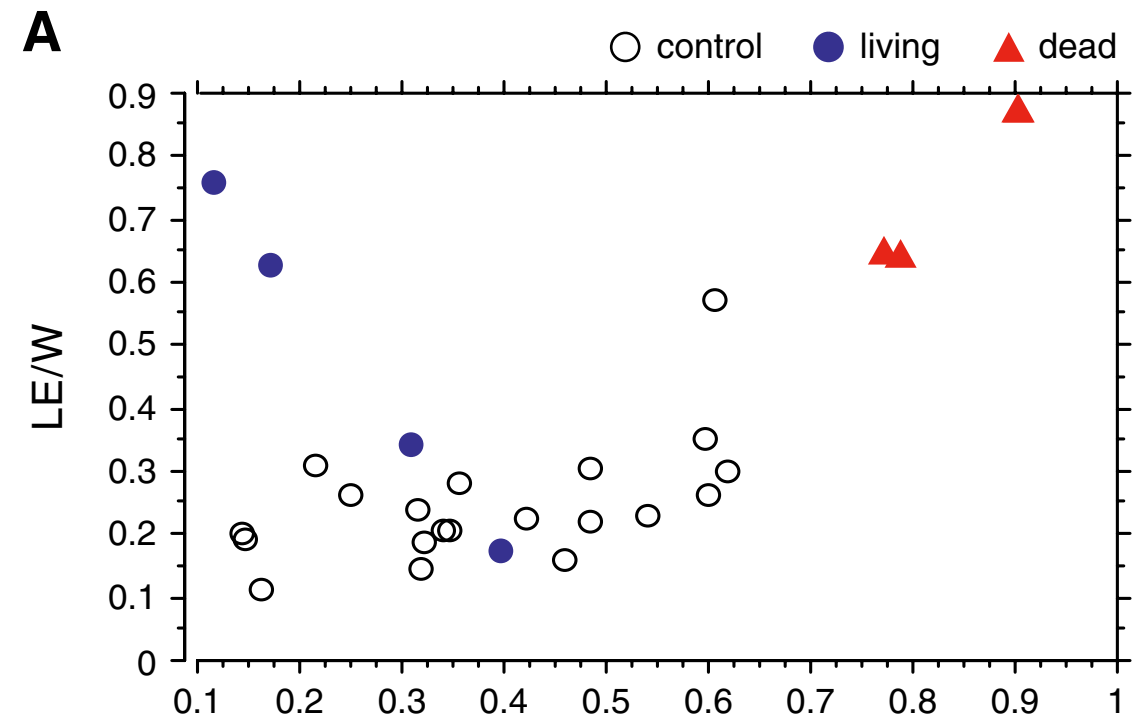

B

\section{RE/W}

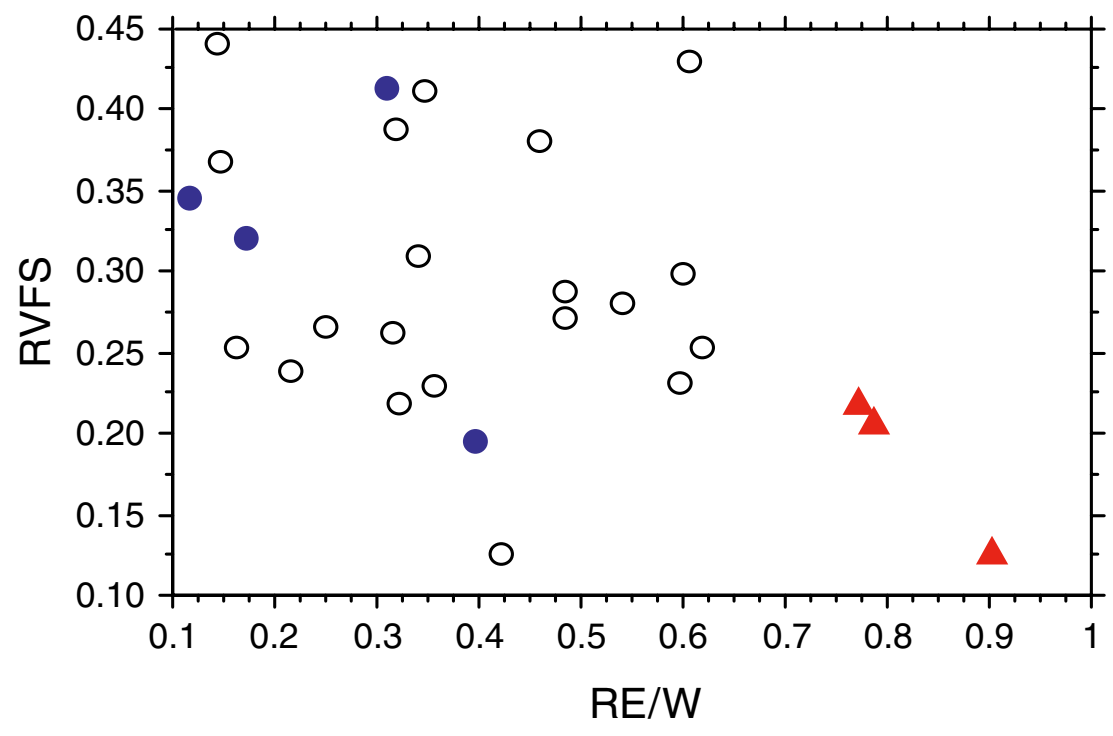

Figure 3. Correlations between the ratio of the thickness of the right and left ventricular endocardium to that of the whole ventricular wall in end-diastole (RE/W, LE/W) and between RE/W and right ventricular fractional shortening (RVFS). White circle, control; Blue dot, living; Red triangle, dead. (A) Correlation between RE/W and LE/W. Patients from the dead group located in the right upper quadrant showed high values for both RE/W and LE/W. Only LE/W was high in patients from the living group. (B) Correlation between RE/W and RVFS. Patients from the dead group located in the lower right quadrant had a high RE/W and a low RVFS.

ever, only LE/W was high in the living group. In addition, Figure 3B shows the association between RE/W and RVFS. Patients in the dead group (located in the lower right quadrant) had high RE/W and low RVFS.

\section{Maternal Autoantibody}

Figure 4 shows the correlation between the prognosis and the titer of each autoantibody. Mothers of all patients were serologically positive for antibodies specific for $52-\mathrm{kDa}$ and $60-\mathrm{kDa}$ antigens. Antibodies to the 52-kDa SSA and the 60$\mathrm{kDa}$ SSA antigens did not differ between the living and dead groups. There was no correlation between the titer of each autoantibody and fetal echocardiographic data.

\section{Discussion}

Isolated $\mathrm{CCHB}$ is frequently found in the offspring of mothers who have anti-SSA autoantibodies. Immune reactivity due to maternal antibodies is implicated in the development of congenital heart block. ${ }^{7}$ In previous reports, the mortality rates of CCHB patients varied from $8 \%$ to $19 \%, 5,8$ and mortalities were largely attributable to cardiac failure in the neonatal period. ${ }^{8}$ Patients with CCHB having fetal, neonatal, or childhood diagnosis had a mortality of $43 \%, 6 \%$, and $0 \%$, respectively. ${ }^{8}$ Increased mortality risk was associated with a fetal diagnosis of CCHB, hydrops fetalis, EFE, and delivery at less than 32 weeks. ${ }^{8}$ Cardiomyopathy, such as EFE and dilated cardiomyopathy, is particularly associated with high mortality rates in CCHB patients. ${ }^{3,4}$ Nield et al also reported that EFE accounted for over $80 \%$ of deaths. ${ }^{9}$ In our study, all patients were diagnosed with $\mathrm{CCHB}$ during the fetal period and the mortality rate was as high as $42 \%$, which is similar to that given in previous reports. All 5 deaths in our study were associated with EFE and hydrops fetalis, indi- 
A

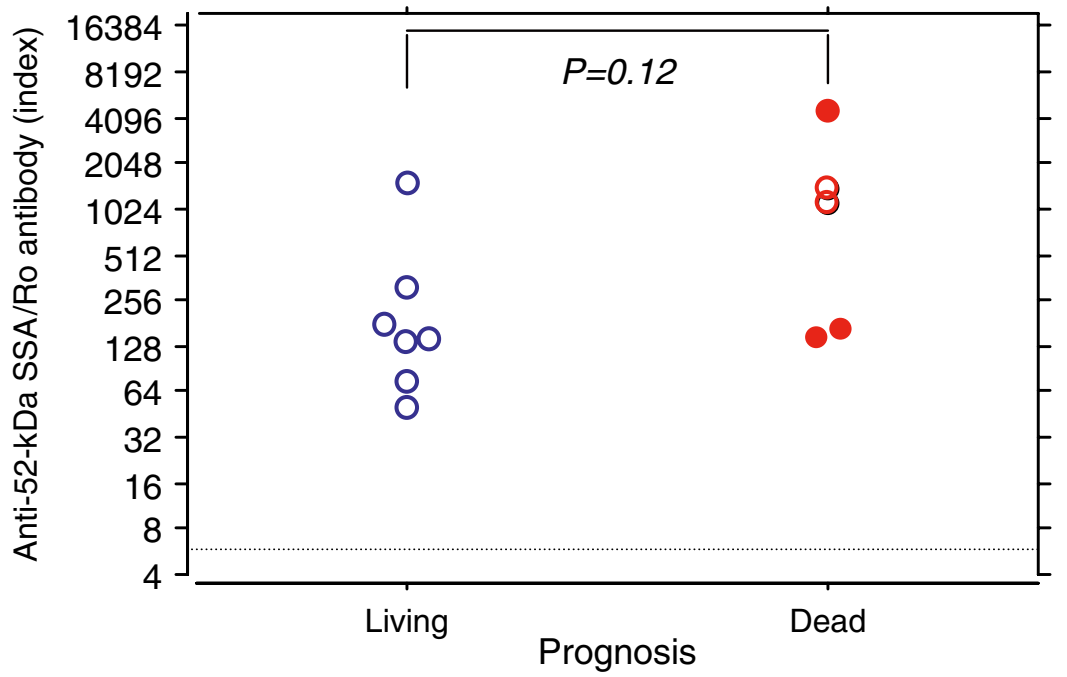

B

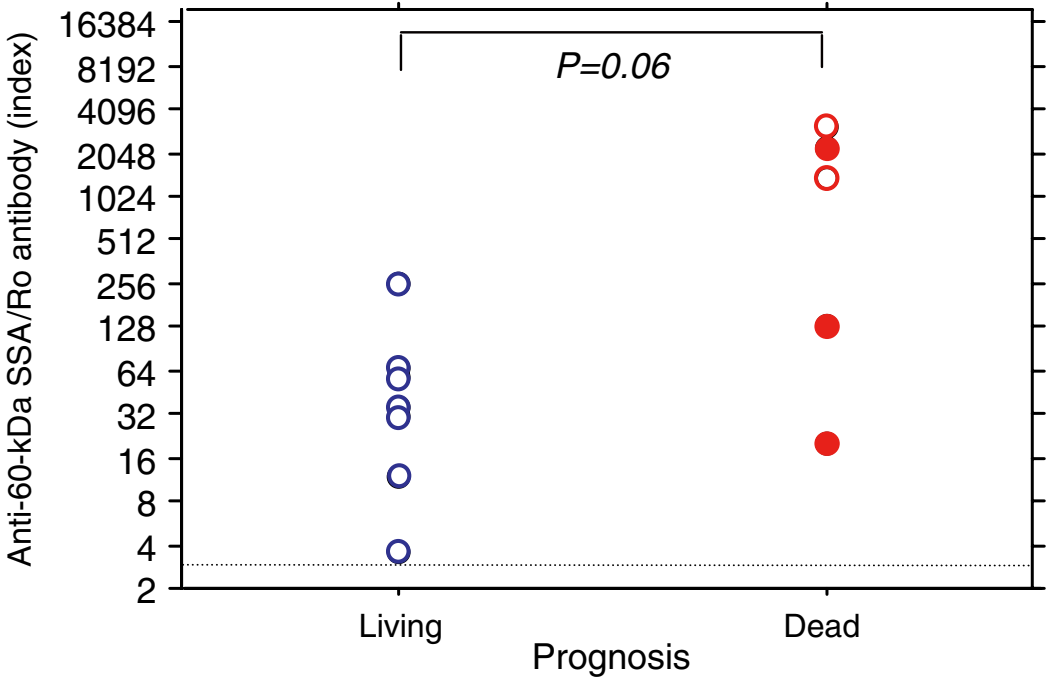

Figure 4. Titers of anti-52-kDa SSA antibody and anti-60-kDa SSA antibody. Scatter plots show the association between prognosis and titers of anti-52-kDa SSA antibody and anti-60-kDa-SSA antibody, respectively. (A) Anti-52-kDa SSA antibody titer was not significantly different between living and dead $(P=0.12)$. (B) Anti-60-kDa SSA antibody was not significantly different between the living and dead groups $(P=0.06)$. Data were analyzed using the Mann-Whitney $U$-test. A P value of $<0.05$ was considered significant. Blue circle, living; Red circle, late death; Red dot, prenatal or neonatal death.

cating that EFE and hydrops fetalis were the major negative prognostic factors for CCHB.

We were able to review the fetal echocardiographic findings in 7 of the 12 patients, including 3 (cases $9,11,12$ ) of the 5 who had died. Fetal echocardiography showed a thick and hyperintense inner layer of the LV and RV, which was thought to be due to myocardial changes. During histological examination of the ventricular wall, the myocardium showed an increase in elastic fibers and the endocardium showed thickening (Figure 5). Fetal echocardiography showed that RE/W in the dead group was significantly higher than that in the living group. Nield et al also reported that prenatally diagnosed EFE cases predominantly involved the RV and had severe RV dysfunction. ${ }^{4}$ In cases diagnosed postnatal, however, EFE predominantly involved the LV and tended to be most prominent along the LV free wall and the interventricular septum. Results showed that only a high LE/W was observed in the living group, whereas a high RE/W and a low RVFS was observed in the dead group. These results suggest on the 1 hand that severe myocardial damage in the RV during the fetal period leads to RV dysfunction and intrauter- ine or neonatal death. On the other hand, myocardial damage that is restricted to the $\mathrm{LV}$ does not cause perinatal death. This might be because the fetal RV is the driving pump for systemic circulation and the RV endocardium receives a stronger afterload, ${ }^{10}$ the RV has less oxygenation, or the RV has a different developmental process of myocardial compaction from that in the LV.

It has been suggested that EFE may be secondary to an autoimmune process similar to CCHB ${ }^{4}$ The amount of maternal anti-SSA antibodies correlates with the risk of cardiac complications. ${ }^{11}$ Antibodies to the $60-\mathrm{kDa}$ and $52-\mathrm{kDa}$ SSA antigens are uniformly present in neonatal lupus erythematosus (NLE), ${ }^{3}$ and although antibodies to the $60-\mathrm{kDa}$ SSA antigen are very specific for NLE, antibodies to the $52-\mathrm{kDa}$ SSA protein have been shown to cause heart block in an isolated perfused rabbit heart model of NLE. ${ }^{12}$ Antibodies to the 52-kDa SSA antigen, especially the p200 epitope, have been suggested as a relevant marker in evaluating the risk for fetal CCHB. ${ }^{13,14}$ Some investigators have reported that cardiac immunoglobulin deposition in CCHB is associated with maternal anti-SSA autoantibodies. ${ }^{15}$ Our results showed 

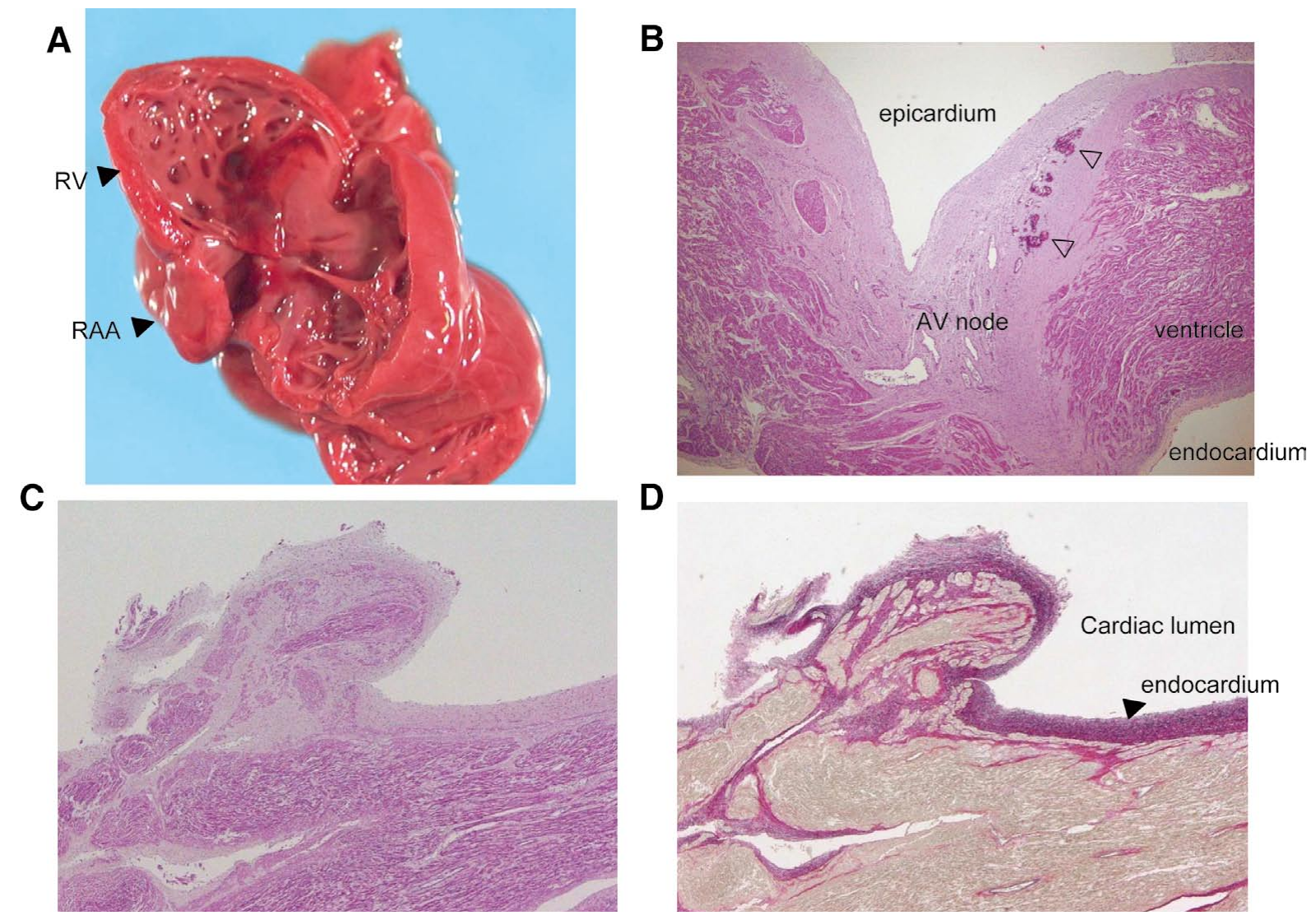

D

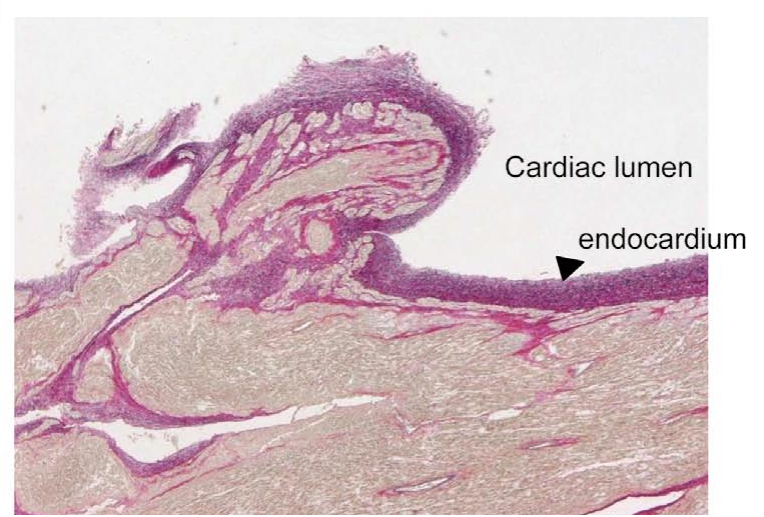

Figure 5. Histological findings from samples of myocardium from case 12. (A) Macroscopic findings. (B) Atrioventricular node (H\&E). (C) Working muscle (H\&E). (D) Elastica van Gieson. Cardiac fiber hypertrophy is indistinct, but the endocardium is thickened with an increase in elastic fibers. AVN areas are degenerated with fibrosis and calcification (open arrows). RV, right ventricle; RAA, right atrial appendage; AVN, atrioventricular node. ${ }^{10}$

a high titer of both the maternal SSA protein and of the 52-kDa and 60-kDa anti-SSA antibodies in CCHB patients. Furthermore, immunopathologic findings showed deposition of immunoglobulin in a patient who died. ${ }^{16}$ These results indicate that a high titer of maternal anti-SSA antibody might be associated with the onset of CCHB, but they do not prove that a higher titer leads to EFE. An immune reaction is necessary for the development of EFE, but other factors such as circulatory load, infection, or genetic inheritance could be implicated. A recent study has described the anti-SSA antibody from mothers binding to the L-type calcium channel in the fetus, which leads to calcium dysregulation and subsequent apoptosis in a small number of patients with susceptibility genes such as tumor necrosis factor- $\alpha$ or transforming growth factor- $\beta$ polymorphisms. ${ }^{17}$ Antibody deposition induces the spread of inflammatory reaction and subsequent fibrosis and/or calcification. Some patients born to mothers with the autoantibodies are diagnosed prenatally with EFE, and others are diagnosed with EFE several years after birth. Development of late-onset cardiomyopathy or EFE may be due to progression of the perinatally impaired myocardium, which requires a secondary trigger such as viral infection or genetic predisposition, and may be induced by pacing or metabolic disorders. ${ }^{9}$ In our study, 1 patient (case 8) was diagnosed with EFE at 6 years after birth and underwent pacemaker implanta- tion. The other (case 6) was diagnosed with INVM at 8 years after birth and showed myocardial damage in only the LV during the fetal period. These patients did not show a deterioration of cardiac performance during the fetal or neonatal periods; however, deterioration of cardiac performance or development of arrhythmia occurred several years after pacemaker implantation. ${ }^{18}$ Because the pacemaker may further deteriorate myocardial damage that had occurred during the fetal period, it is important to continue observation for myocardial abnormalities over a long time period in CCHB patients.

\section{Study Limitations}

The method of measuring some parameters by fetal echocardiography was limited because the image quality of some scans was fuzzy (the videotape recording quality affected the measurement). The small number of cases was another limitation. Further studies using a larger number of cases will be considered.

\section{Conclusions}

Echocardiography data were reviewed for 12 patients with CCHB with or without EFE. We also measured maternal anti-SSA antibody levels in the mothers of these 12 patients. 
An immune reaction is necessary to develop EFE, but other factors such as circulatory load, infection, or genetic inheritance could also be required. Development of EFE could be a major negative prognostic factor for $\mathrm{CCHB}$. Myocardial damage predominantly in the RV was related to the outcome of CCHB associated with EFE.

\section{References}

1. Reed BR, Lee LA, Harmon C, Wolfe R, Wiggins J, Peebles C, et al. Autoantibodies to ss-a/ro in infants with congenital heart block. J Pediatr 1983; 103: 889-891.

2. Scott JS, Maddison PJ, Taylor PV, Esscher E, Scott O, Skinner RP. Connective-tissue disease, antibodies to ribonucleoprotein, and congenital heart block. N Engl J Med 1983; 309: 209-212.

3. Taylor-Albert E, Reichlin M, Toews WH, Overholt ED, Lee LA Delayed dilated cardiomyopathy as a manifestation of neonatal lupus: Case reports, autoantibody analysis, and management. Pediatrics 1997; 99: $733-735$

4. Nield LE, Silverman ED, Taylor GP, Smallhorn JF, Mullen JB, Silverman $\mathrm{NH}$, et al. Maternal anti-ro and anti-la antibody-associated endocardial fibroelastosis. Circulation 2002; 105: 843-848.

5. Jaeggi ET, Hamilton RM, Silverman ED, Zamora SA, Hornberger LK. Outcome of children with fetal, neonatal or childhood diagnosis of isolated congenital atrioventricular block: A single institution's experience of 30 years. J Am Coll Cardiol 2002; 39: 130137.

6. Sahn DJ, Lange LW, Allen HD, Goldberg SJ, Anderson C, Giles $\mathrm{H}$, et al. Quantitative real-time cross-sectional echocardiography in the developing normal humam fetus and newborn. Circulation 1980; 62: $588-597$.

7. Julkunen H, Kaaja R, Wallgren E, Teramo K. Isolated congenital heart block: Fetal and infant outcome and familial incidence of heart block. Obstet Gynecol 1993; 82: 11-16.

8. Buyon JP, Hiebert R, Copel J, Craft J, Friedman D, Katholi M, et al. Autoimmune-associated congenital heart block: Demographics, mortality, morbidity and recurrence rates obtained from a national neonatal lupus registry. J Am Coll Cardiol 1998; 31: 1658-1666.
9. Nield LE, Silverman ED, Smallhorn JF, Taylor GP, Mullen JB, Benson LN, et al. Endocardial fibroelastosis associated with maternal anti-ro and anti-la antibodies in the absence of atrioventricular block. J Am Coll Cardiol 2002; 40: 796-802.

10. Ciurzynski M, Lichodziejewska B, Tomaszewski A, PiotrowskaKownacka D, Kownacki L, Rymarczyk Z, et al. Biventricular noncompaction associated with left ventricular systolic and diastolic dysfunction and severe pulmonary hypertension in a young man. Circ J 2009; 73: 2163-2165.

11. Jaeggi E, Laskin C, Hamilton R, Kingdom J, Silverman E. The importance of the level of maternal anti-ro/ssa antibodies as a prognostic marker of the development of cardiac neonatal lupus erythematosus a prospective study of 186 antibody-exposed fetuses and infants. J Am Coll Cardiol 2010; 55: 2778-2784.

12. Viana VS, Garcia S, Nascimento JH, Elkon KB, Brot N, Campos de Carvalho AC, et al. Induction of in vitro heart block is not restricted to affinity purified anti-52 kda ro/ssa antibody from mothers of children with neonatal lupus. Lupus 1998; 7: 141-147.

13. Salomonsson S, Dorner T, Theander E, Bremme K, Larsson P, Wahren-Herlenius M. A serologic marker for fetal risk of congenital heart block. Arthritis Rheum 2002; 46: 1233-1241.

14. Strandberg L, Winqvist O, Sonesson SE, Mohseni S, Salomonsson S, Bremme K, et al. Antibodies to amino acid 200-239 (p200) of ro52 as serological markers for the risk of developing congenital heart block. Clin Exp Immunol 2008; 154: 30-37.

15. Lee LA, Coulter S, Erner S, Chu H. Cardiac immunoglobulin deposition in congenital heart block associated with maternal anti-ro autoantibodies. Am J Med 1987; 83: 793-796.

16. Iida $\mathrm{M}$, Inamura $\mathrm{N}$, Takeuchi $\mathrm{M}$. Newborn infant with maternal anti-ssa antibody-induced complete heart block accompanying cardiomyopathy. Circ J 2006; 70: 147-149.

17. Wahren-Herlenius M, Sonesson SE. Specificity and effector mechanisms of autoantibodies in congenital heart block. Curr Opin Iттиnol 2006; 18: 690-696.

18. Takemoto Y, Hasebe H, Osaka T, Yokoyama E, Kushiyama Y, Suzuki T, et al. Right ventricular septal pacing preserves long-term left ventricular function via minimizing pacing-induced left ventricular dyssynchrony in patients with normal baseline qrs duration. Circ J 2009; 73: 1829-1835. 